\title{
An Analysis of the Distribution of Volumes amongst Spores of the Cellular Slime Mould Dictyostelium discoideum
}

\author{
By M. J. SACKIN AND J. M. ASHWORTH \\ M.R.C. Microbial Systematics Unit and Department of Biochemistry, \\ School of Biology, Leicester University, Leicester, LEI 7 RH
}

(Accepted for publication 30 August 1969)

\begin{abstract}
SUMMARY
The mean volume of a diploid spore population is twice that of a haploid spore population; it is assumed that the mean volume of an aneuploid spore population is a similar reflexion of its ploidy. The distribution of volumes amongst a population of spores of Dictyostelium discoideum was analysed in terms of frequency of occurrence of diploid, aneuploid and haploid cells; in a wild-type strain (NC-4) these cell types were present in the ratio 8:3:89. It is suggested that 'metastable' strains are mutants in which this ratio has been altered.
\end{abstract}

The life cycle of the cellular slime mould Dictyostelium discoideum is divided into two mutually exclusive phases. In the first, feeding, phase the organism exists as solitary amoeboid cells and in the second, differentiation, phase these hitherto solitary amoeboid cells associate and, without any change in number, produce a fruiting body consisting of spore and stalk cells. The spores germinate in a suitable environment to give amoeboid cells, each spore giving one myxamoeba. The stalk cells are nonviable. Recent studies of mutants derived from a haploid strain of $D$. discoideum have shown that genetic exchange can occur at a low frequency during this life cycle (Loomis \& Ashworth, 1968; Sinha \& Ashworth, 1969; Loomis, 1969). Segregation occurs from heterozygous diploids in a manner which suggests haploidization via stepwise and random chromosome loss, rather than by meiotic division. Direct chromosome counts during the period of haploidization support this suggestion (Sinha \& Ashworth, I969). It appears, therefore, that $D$. discoideum has a parasexual cycle of the type described by Pontecorvo (1956) for Aspergillus and shown in Fig. 4. The genotype of the spores must therefore be identical with the genotype of the myxamoebae from which they are derived. There should thus exist, in any population of spores, diploid, haploid and aneuploid genotypes. Sussman \& Sussman (1962) suggested that the ploidy of a spore could be correlated with its size. They reported that the mean major diameter of a diploid spore was about one and a half times the mean major diameter of a haploid spore.

We report here that the mean volume of a diploid spore population is twice that of a haploid spore population and that the distribution of volumes amongst a spore population can therefore be analysed in terms of the frequency of occurrence of haploids and diploids. Such an analysis also enables the frequency of occurrence of aneuploids to be estimated. The nature of the distribution of ploidy types in the population can be calculated if it be assumed that very few non-viable aneuploid 
myxamoebae produce spores and that when aneuploids are produced from diploids each chromosome has an equal and constant chance of being lost at each division. This analysis predicts the occurrence, and describes the nature, of strains isolated by Sussman \& Sussman (I962) and called by them 'metastable'.

\section{METHODS}

Organisms. Dictyostelium discoideum NC-4 is a haploid strain isolated by Raper (1935). Dictyostelium discoideum $\mathrm{H}-\mathrm{I}$ is a diploid, yellow pigment producing, strain isolated by Sussman \& Sussman (I962) from a mixed culture of a mutant strain which produces a brown pigment and another mutant strain which produces a white pigment. Both strains were the gift of Dr M. Sussman (Brandeis University, Waltham, Mass., U.S.A.).

Volume determination. Myxamoebae were grown in association with Aerobacter aerogenes on agar plates and allowed to form fruiting bodies as described by Sussman (1966). Spores were collected from agar plates $36 \mathrm{~h}$ after the disappearance of the bacterial lawn. They were collected in a drop of $0.9 \%$ sterile $\mathrm{NaCl}$ in a platinum loop and dispersed in $60 \mathrm{ml}$. of sterile $0.9 \% \mathrm{NaCl}$. Volume measurements were made by using a Coulter Counter Model B with a $30 \mu \mathrm{m}$. aperture. This aperture size is suitable for the analysis of particles whose mean diameters fall in the range 2 to $20 \mu \mathrm{m}$. The mean major diameters of $D$. discoideum spores are in the range 7 to $10 \mu \mathrm{m}$.; similar results were obtained when a $100 \mu \mathrm{m}$. aperture was used. Measurements were made with the following settings on the instrument: $\mathrm{I} /$ amplification $=\mathrm{I} /$ aperture current $=4$, upper threshold setting $=20$ and locked with the lower threshold setting. Measurements were taken at the odd numbered settings of the lower threshold dial. Samples $\left(0.5 \mathrm{ml}\right.$.) of the spore suspensions at $\leqslant 3 \times 10^{4}$ spores $/ \mathrm{ml}$. were counted at each setting, this corresponds to a counting rate of $<10^{4}$ spores $/ \mathrm{ml}$. Harvey \& Marr (I966) showed that at counting rates greater than this the unmodified Coulter Counter gives seriously inaccurate volume distributions. The spore suspensions were stirred continuously during the measurements which are expressed in terms of the lower threshold setting of the instrument. By using spherical puff ball spores of mean diameter $3.6 \mu \mathrm{m}$. as a standard it was found that Io such units correspond to a particle of volume $42 \cdot 5 \mu \mathrm{m}^{3}$.

\section{RESULTS}

The distribution of volumes amongst a population of spores of the diploid strain H-I $(n=\mathrm{I} 4)$ of Dictyostelium discoideum is shown in Fig. I. In this, and subsequent, distributions the particles with a volume $<4$ are disregarded. This part of the distribution is caused by dust, particles from the sporangium etc., and not spores. Spores from strain H-I were plated out clonally with Aerobacter aerogenes; one white and one brown clone were picked from amongst the predominantly yellow (H-I) clones. The spores from these strains were transferred to a fresh growth plate, allowed to germinate, the myxamoebae allowed to grow and the resulting spores isolated and analysed. The results of an analysis of the white spores are shown in Fig. I. The size distribution of the spores prepared from the brown clone was like that of the white spore distribution, with a mean of II 0 . Similar strains have been isolated from H-I by Sussman \& Sussman (I963) who have shown, cytologically, that they are haploid $(n=7)$. The mean 
volume of the diploid spore population (22) is exactly twice that of the two haploid spore populations derived therefrom (both II).

Spores from strain H-I were also subcultured without cloning. There was a progressive change in the volume distributions after each mass sub-culture (Fig. 2). In our hands strain H-I, although much more stable than other diploids (Sinha \& Ashworth, 1969), could only be kept by repeated isolation of yellow spore-forming clones. Eventually, after frequent mass sub-culturing, the stock was 'lost' and became predominantly haploid. The term 'stable' as applied to strain H-I is thus a relative term.

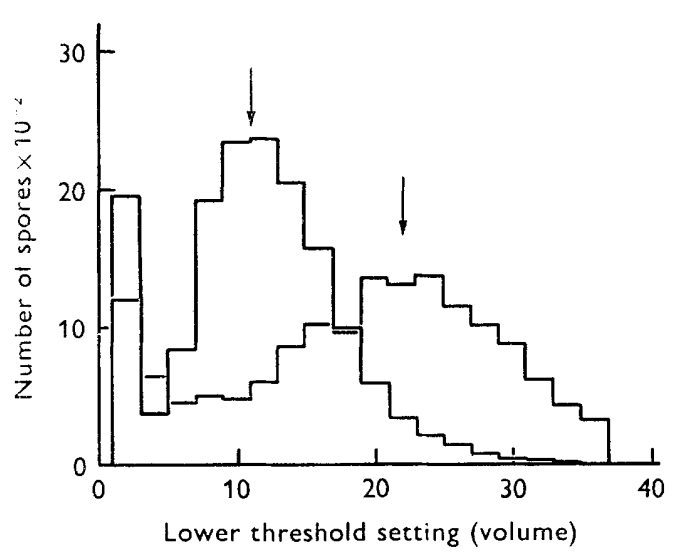

Fig. I

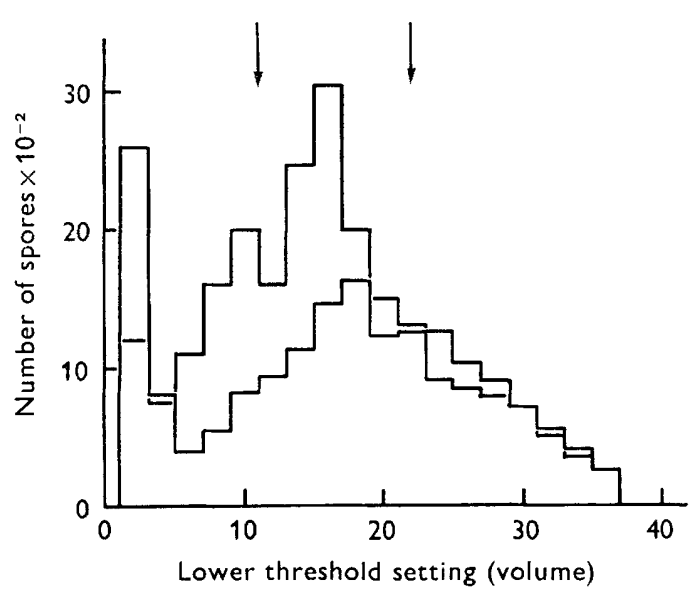

Fig. 2

Fig. I. Distribution of volumes amongst spore populations derived from the diploid stock $\mathrm{H}-\mathrm{I}$ (mean 22) and from a haploid stock derived from H-I (mean II). The arrows mark the means.

Fig. 2. Distribution of volumes amongst spore populations derived from a clone of the diploid stock $\mathrm{H}$-I after 3 and 6 non-clonal sub-cultures. The arrows mark the mean volumes of the haploid and diploid populations.

The distribution of volumes amongst a population of spores from the haploid strain NC-4 is shown in Fig. $3 a$ : the distribution is similar in shape to that of the haploid stocks derived from the diploid stock $\mathrm{H}-\mathrm{I}$. The mean volume of $\mathrm{NC}-4$ (I2.5) was, however, slightly but significantly larger than the mean volumes found for the white and brown haploid stocks isolated from $\mathrm{H}-\mathrm{I}$ ( $\mathrm{II} \cdot 0)$, in agreement with the observations of Sussman \& Sussman (I962). This volume distribution (Fig. $3 a$ ) is a normal distribution curve skewed to large volumes. We have shown that the volume of a spore can be correlated with its chromosome number and thus this distribution can be analysed in terms of contributions from haploid and diploid spores. At low volumes (below 12) the contribution of the haploid spores to the population will predominate. By using the techniques described by Harris (1968) the mean and standard deviation of the haploid population were calculated and the appropriate normal distribution fitted to the experimental curve. Subtraction of this distribution from the experimental distribution (Fig. $3 b$ ) will leave the contribution of the nonhaploid spores to the total. At large volumes (above 25) the contribution of the diploid spores will predominate. By using the same techniques, a normal distribution 
curve can be fitted to, and subtracted from, the experimental curve. A considerable amount of the distribution remained unaccounted for after these procedures.

Sinha \& Ashworth (1969) showed by using genetic techniques that diploid stocks of Dictyostelium discoideum haploidized via transient aneuploids; the parasexual cycle they suggested is shown in Fig. 4. If the exact relationship between spore volume and chromosome number which we have shown to exist for haploid and diploid spores also holds for aneuploid spores then that portion of the total volume distribution of strain $\mathrm{NC}-4$ which is unaccounted for after removal of the haploid and diploid contributions must be ascribed to aneuploid spores. The ratio of haploids:aneuploids: diploids calculated from the distribution shown in Fig. 3 is $89: 3: 8$.

(a)

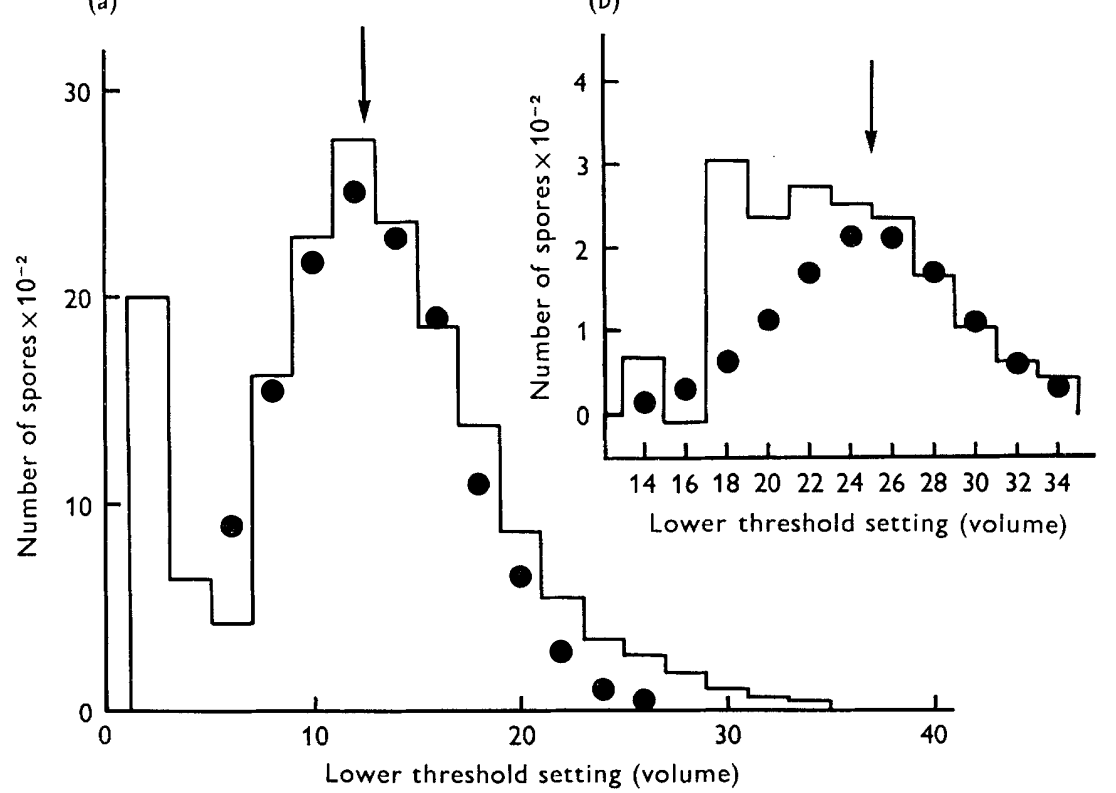

Fig. 3. (a) Distribution of volumes amongst a spore population derived from $D$. discoideum NC-4. normal distribution with $\bar{x}=12.5$ and S.D. $=4.5$ fitted to the observation distribution. (b) Volume distribution remaining after subtraction of the calculated normal distribution from the data of Fig. $3(a)$. Normal distribution with $\bar{x}=25$ and s.D. $=4 \cdot 5$ fitted to these volumes. In both cases the arrows mark the mean volumes.

\section{Analysis of the aneuploid population}

Aneuploid spores may be described as $A_{8}, A_{9}, A_{10}, A_{11}, A_{12}$ or $A_{13}$ classes according to the number of chromosomes they contain. Each class may be further subdivided into several different chromosome configurations. There will thus be seven different chromosome configurations for the $A_{8}$ class. An aneuploid spore is derived from an aneuploid myxamoeba which is, in turn, derived either from an aneuploid myxamoeba containing more chromosomes or a diploid myxamoeba (Fig. 4). The mechanism whereby chromosomes are lost at division is unknown but it would seem plausible that the chance of any particular chromosome being lost from a diploid or aneuploid at any one division is constant. If a diploid (or aneuploid) loses two identical chromosomes then it will presumably be non-viable even though it may still have more than 
seven chromosomes. Some of these non-viable aneuploids may be capable of forming spores but the chances of their doing so seem slight; even if they did they would comprise a very small fraction of the total aneuploid population, being diluted out by the viable aneuploids. We can thus neglect the contribution made by the nonviable aneuploids (if any) to the total spore population. Two mechanisms can be suggested whereby a new aneuploid myxamoeba is produced. One possibility is that the chromosomes of an aneuploid replicate and these replicated chromosomes are then completely but unequally shared between two, or perhaps more, daughters. The

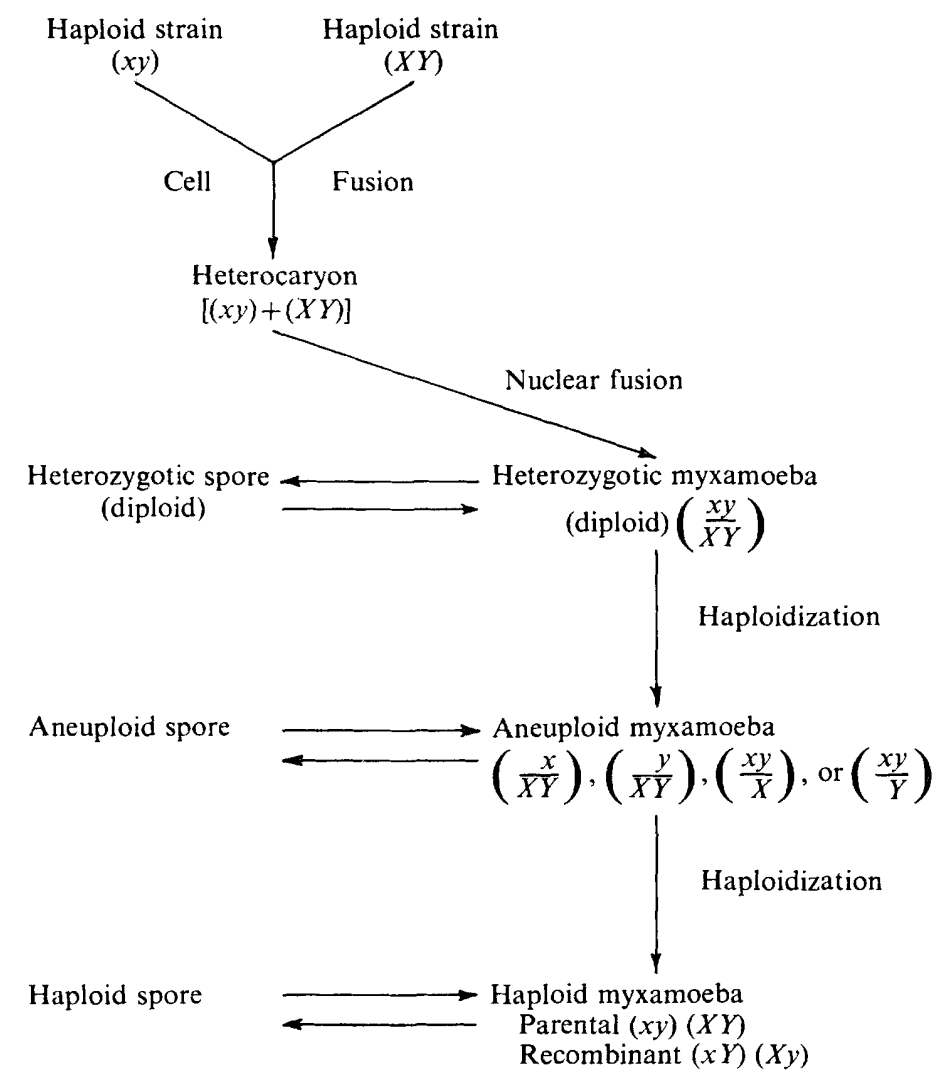

Fig. 4. The parasexual cycle of $D$. discoideum. $x / X$ and $y / Y$ represent two unlinked genes and the genotype of the various kinds of nuclei are enclosed in parentheses (Sinha \& Ashworth, 1969).

other possibility is that no replication occurs but that within one division time an aneuploid divides to give either another aneuploid with fewer chromosomes, or a haploid and the extra chromosomes (which will be less than seven) are degraded or ejected.

The first possibility is difficult to handle mathematically. It is also unlikely in the case of Dictyostelium discoideum where the aneuploids are unstable (Sinha \& Ashworth, 1969; Loomis, 1969). The second possibility is easier to handle mathematically and is in accord with the known instability of aneuploids. These two possibilities are not mutually exclusive. It is possible to imagine partial replication to occur before 
division, and this might explain the rare tripolar mitotic figures which have been seen by us and by Sussman \& Sussman (I963). However, we shall disregard partial replication here (see Discussion).

Let $P$ be the probability that any given chromosome disappears during one division cycle. Suppose that the equilibrium frequencies of $A_{k}$ cells are $P_{k}(k=8,9, \ldots$, I4). During one division cycle any $k$-aneuploid will have been derived from an aneuploid of class $A_{j}$, say, with a higher or equal ploidy number. Thus $j$ may equal $k, k+\mathrm{I}, \ldots$, or I4. For all pairs of $k$ and $j(8 \leqslant k \leqslant j \leqslant 14)$ we shall derive an expression for the proportion of $A_{k}$ cells which derive from $A_{j}$ cells during one division cycle. This proportion will be a multiple of $\boldsymbol{P}_{j}$ and will also depend on $\boldsymbol{P}$. Thus $\boldsymbol{P}_{k}$ will be a linear combination of $P_{k}, P_{k+1}, \ldots, P_{14}$. (Since we are dealing with equilibrium conditions $P_{k}$ does not vary with time, so that a proportion $P_{k}$ of $A_{k}$ cells are formed during one division time.) We shall then have a set of simultaneous linear equations in $P_{k}(k=8,9, \ldots$, I4) which can be solved.

We shall use notation of the type (l $\left.\begin{array}{llllll}\hline & 2 & \text { I } & 2 & 2 & 2\end{array}\right)$ for a typical chromosome configuration; here there is a single representative of the first and fourth chromosomes and two representatives of the others.

The probability that a diploid cell $\left(\begin{array}{llllll}2 & 2 & 2 & 2 & 2 & 2\end{array}\right)$ changes into an $A_{13}$ cell of con-

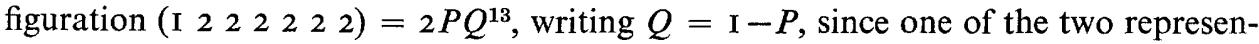
tatives of the first chromosome is lost, and the other I3 chromosomes are not lost. There are

$$
\left.7 \text { (i.e. }\left(\begin{array}{l}
7 \\
\mathrm{r}
\end{array}\right)\right) A_{13} \text { configurations. }
$$

Hence

$$
\text { prob }\left(A_{14} \text { changes to } A_{13}\right)=2 \times\left(\begin{array}{l}
7 \\
\mathrm{I}
\end{array}\right) P Q^{13} \text {. }
$$

Similarly,

$$
\text { prob }\left(A_{14} \text { changes to } A_{12}\right)=2^{2} \times\left(\begin{array}{l}
7 \\
2
\end{array}\right) P^{2} Q^{12} \text {, }
$$

where $A_{12}$ is used to denote a viable I2-aneuploid and similarly for the other $A$ 's.

In general

$$
\text { prob }\left(A_{14} \text { changes to } A_{k}\right)=2^{14-k}\left(\begin{array}{c}
7 \\
14-k
\end{array}\right) P^{14-h} Q^{k}(k=8,9, \ldots, \text { I } 4) .
$$

We may generalize further to give:

$$
\text { prob }\left(A_{j} \text { changes to } A_{k}\right)=2^{j-k}\left(\begin{array}{l}
j-7 \\
j-k
\end{array}\right) P^{j-k} Q^{k} \text {. }
$$

Thus the proportion ${ }^{-}$of total cells which change from $A_{j}$ to $A_{k}$ during one division cycle is

$$
2^{j-k} P_{j}\left(\begin{array}{c}
j-7 \\
j-k
\end{array}\right) P^{j-k} Q^{k}
$$

Since all $k$-aneuploids derive from $j$-aneuploids $(j=k, k+\mathrm{I}, \ldots, \mathrm{I} 4)$, we have

for $k=8,9, \ldots, \mathrm{I} 3$.

$$
P_{k}=\sum_{j=k}^{14} 2^{j-k} P_{j}\left(\begin{array}{c}
j-7 \\
j-k
\end{array}\right) P^{j-k} Q^{k}
$$

Solving equation (I) for various values of $P$ it appears that the relative frequencies $P_{k}$ of aneuploids and diploids are an extremely sensitive function of $P$ (Fig. 5). The experimentally determined value for the ratio diploid:aneuploid is approximately $2 \cdot 4$ and is given by $P_{14} /\left(P_{8}+P_{9}+\ldots+P_{13}\right)$ in our notation. From Fig. 6 it is seen that this ratio 
is given by $P \simeq 0.35$. Solving equation (I) with this value of $P$ we obtain the following values for the relative equilibrium frequencies of the various classes of aneuploid, assuming that the frequency of diploids remains constant: $P_{8} / P_{14}=0.0453$; $P_{9} / P_{14}=0.0926 ; P_{10} / P_{14}=0.1245 ; P_{11} / P_{14}=0.1090 ; P_{12} / P_{14}=0.0593 ; P_{13} / P_{14}=$ 0.0182 .

The mean value $v_{k}$ of the spore volume distribution corresponding to the aneuploid class $A_{k}$ is given by

$$
v_{k}=\frac{25 k}{\mathrm{I} 4}
$$

on the assumption that $v_{k}$ is proportional to the ploidy number $k$. It is not possible to calculate the standard deviation corresponding to this mean, but we take this to be equal to the standard deviations of the haploid and diploid populations (both 4.5 ) and

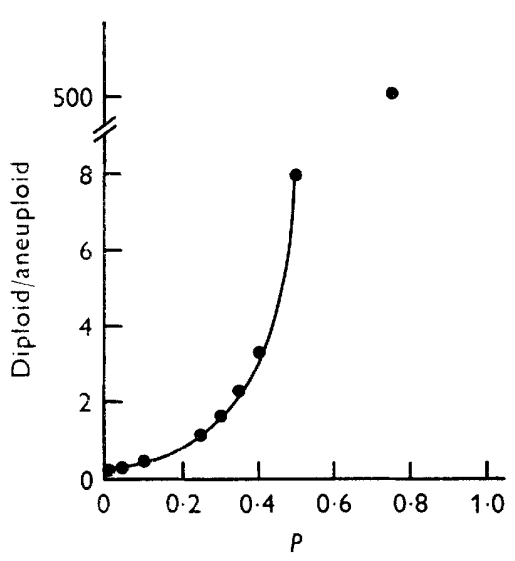

Fig. 5

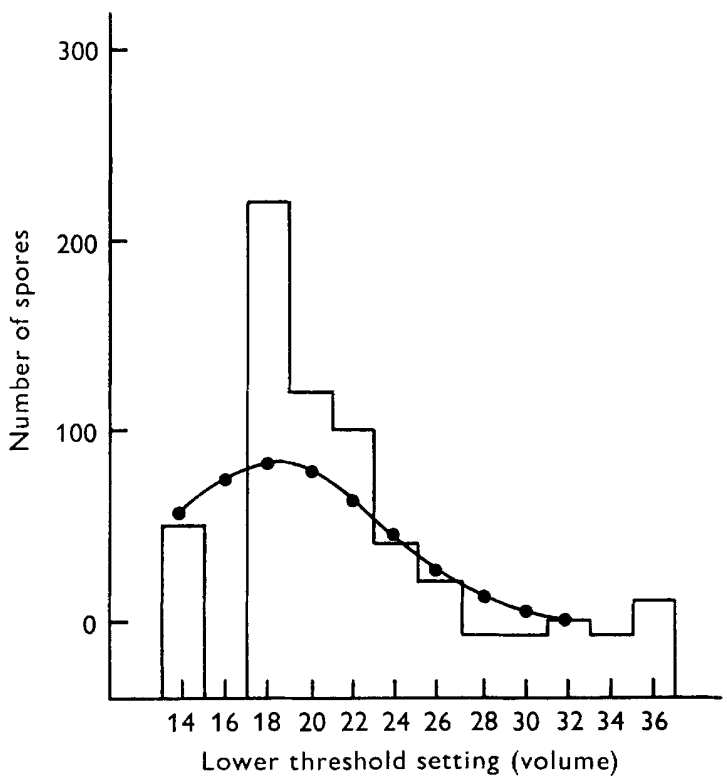

Fig. 6

Fig. 5. Dependence of the diploid:aneuploid ratio on the value of $P$, the probability that any one chromosome will be lost at any one division.

Fig. 6. Observed (histogram) distribution of spore volumes of D. discoideum NC-4 remaining after subtraction of the haploid and diploid contributions and calculated distribution of volumes (O) assuming that these spores are aneuploids with $P=0.35$ and s.D.s $=4.5$.

assume that it is normally distributed. Using these assumptions and the values of the relative frequencies of the aneuploid classes we obtain a calculated volume distribution for the aneuploids (Fig. 6). Since the final distribution is relatively unaffected by the exact values taken for the standard deviation and the exact form of each aneuploid class volume distribution this uncertainty has little affect on our calculations.

A change in $P$ not only alters the diploid:aneuploid ratio; it also alters the relative frequency of the various aneuploid classes. Thus at $P=0.35$ the predominant aneuploid class is $A_{10}$. As $P$ tends to zero the predominant aneuploid classes are $A_{12}$ and $A_{13}$; at $P=0.5$ it is $A_{9}$, and for values of $P$ close to $\mathrm{I} \cdot 0$ it is $A_{8}$. It seems reasonable that 
this should be so since, if $P$ is large, chromosomes are lost so quickly that very few aneuploids exist and those that do exist have only one or two extra chromosomes. Conversely, if $P$ is small, chromosomes are lost so slowly that aneuploids are quite frequent and many of them have a large number of extra chromosomes.

\section{DISCUSSION}

Sussman \& Sussman (1963) reported that diploid spores of Dictyostelium discoideum often have an unusual and irregular shape; this was confirmed by Sinha \& Ashworth (I969). It is thus not surprising that it is the mean volume of a spore population which is proportional to the ploidy and not the mean major diameter. It is not unusual to find amongst other fungi (Clutterbuck, 1969) and higher organisms (Noggle, 1946) relationships of this type.

The analysis of volume distributions of the type shown in Fig. 2 in terms of contributions from haploid, diploid and aneuploid spores gives, at best, an approximate answer, because the total number of aneuploid spores present is so small. Any procedure which entails measuring accurately the relatively small difference between two large numbers is bound to be approximate. However, the Coulter Counter can handle, accurately, large numbers of spores in a reasonable time, and, as can be seen in Figs. 3 and 6 , the agreement between the calculated and experimental volume distributions is acceptable. This agreement can be taken to justify the assumptions made in calculating this distribution, namely, $(a)$ aneuploids arise either from diploids or from aneuploids containing a larger number of chromosomes; $(b)$ aneuploids are unstable and the probability $(P)$ that a chromosome will be lost at any one division is a constant; (c) that it can be assumed that there is no chromosome replication before division of an aneuploid.

There is independent, genetic, evidence which justifies some of these assumptions (Loomis \& Ashworth, 1968; Loomis, I969; Sinha \& Ashworth, 1969). Assumption (c) is probably only valid to a first approximation, but it would be difficult to distinguish between partial replication and no replication at all. The occurrence of partial replication can, in part, be allowed for by an alteration in the constant $P$. If it were possible to determine $P$ experimentally then it would be possible to assess the extent of chromosome replication in an aneuploid before its division. Since partial replication will result in an alteration of $P$ and since we have chosen the best value of $P$ to fit our data empirically it is probable that we have allowed, in part, for such replication as occurs.

The frequency of diploids deduced in this way $(8 \%)$ is considerably higher than the frequency of heterozygous diploids as found by Loomis \& Ashworth (I968) after mixing myxamoebae from two genetically different strains $(0.04$ to $2 \%$ ); this suggests that homozygous diploids are formed more readily than heterozygous diploids.

Sussman \& Sussman (1962) described the isolation of mutant strains of Dictyostelium discoideum which had a spore size distribution similar in shape to that of the diploid or haploid spores, but with a mean major diameter between these two extremes; such strains they described as 'metastable'. Cytological investigation showed that these metastable strains contained both diploid and haploid cells, and one strain differed from another in the ratio of haploid:diploid metaphase figures observed. They did not report seeing any aneuploid figures, but these are easily overlooked and 
disregarded, especially since the bacterial debris inside the myxamoebae often stains like the chromosomes.

The size distribution of the spores from metastable strains cannot be explained by assuming these strains to be a mixture solely of haploid and diploid cells. The addition of two normal distributions of the type shown in Fig. I cannot result in another normal distribution with a mean of about 16 and a standard deviation of $4 \cdot 5$. Some of the metastable strains described by Sussman \& Sussman (1962) did, however, have distributions of this type.

We suggest that metastable strains of Dictyostelium discoideum are mutant strains in which the mutational event has affected the value of the constant $P$ which determines the probability that a chromosome will be lost at any one division. It would seem likely that such a mutational event would also affect the haploid:diploid ratio. The aneuploid:diploid ratio is affected by the value of $P$ (Fig. 5), and the predominant aneuploid class in the aneuploid population is also affected by the value of $P$. Thus, by taking appropriate values of $P$ and the haploid:diploid ratio it is possible to construct spore-volume distribution curves which closely resemble those described by Sussman \& Sussman (1962). Distribution curves similar to those of the metastable strains can be obtained by the mass sub-culture of the initially homogenous diploid strain H-I. Here the proportion of diploid:haploid:aneuploid is changing while haploidization proceeds, and distributions of the type shown in Fig. 2 are not equilibrium distributions. They show, however, that our explanation for the spore volume distributions of metastable strains in terms of a change in $P$ and consequent change in diploid: haploid:aneuploid ratio is adequate to account for the experimental observations.

Sussman \& Sussman (I962) reported that many metastable strains arose from cells ('I-cells') which initiated the aggregation phase of the life cycle of Dictyostelium discoideum; they thus represent the first type of cell differentiation shown by this organism. However, metastable strains are also most easily isolated from those cells which do not readily aggregate (Sussman, 1964), a fact difficult to reconcile with their being involved in initiating aggregation. These and other properties of the I-cells become comprehensible in terms of our theory, which would describe such cells as being of a particular aneuploid configuration. The evidence, and implications, for such an assertion will be presented in more detail elsewhere (Ashworth \& Sackin, I969).

We thank the S.R.C. and M.R.C. for financial support.

\section{REFERENCES}

Ashworth, J. M. \& SACKIN, M. J. (1969). Cell differentiation in the cellular slime mould Dictyostelium discoideum: the role of aneuploid cells. Nature, Lond. (in press).

Clutterbuck, A. J. (1969). Cell volume per nucleus in haploid and diploid strains of Aspergillus nidulans. J. gen. Microbiol. 55, $29 \mathrm{I}$.

HARRIS, D. (1968). A method of separating two superimposed normal distributions using arithmetic probability paper. J. Anim. Ecol. 37, 315 .

Harvey, R. J. \& MARr, A. G. (I966). Measurement of size distributions of bacterial cells. J. Bact. 92, 804.

Loomis, W. F. (1969). Temperature sensitive mutants of Dictyostelium discoideum. J. Bact. 99, 65.

Loomis, W. F. \& Ashworth, J. M. (1968). Plaque-size mutants of the cellular slime mould Dictyostelium discoideum. J. gen. Microbiol. 53, I8I. 
NogGLe, G. R. (1946). The physiology of polyploidy in plants. I. Review of the literature. Lloydia $\mathbf{9}$, I 53.

Pontecorvo, G. (1956). The parasexual cycle in fungi. A. Rev. Microbiol. ro, 393.

RAPER, K. B. (1935). Dictyostelium discoideum, a new species of slime mould from decaying forest leaves. J. agric. Res. 50, I35.

Sinha, U. \& Ashworth, J. M. (1969). Evidence for the existence of elements of a para-sexual cycle in the cellular slime mould Dictyostelium discoideum. Proc. Roy. Soc. Lond. B I73, 53I.

Sussman, M. (1964). Isolation of diploid strains of Dictyostelium discoideum from haploid populations. Nature, Lond. 201, 2 I 6.

Sussman, M. (1966). Methods in Cell Physiology, chap. 14. London \& New York: Academic Press.

Sussman, M. \& Sussman, R. R. (1962). Ploidal inheritance in Dictyostelium discoideum: stable haploid, stable diploid and metastable strains. J. gen. Microbiol. 28, 4I 7.

Sussman, M. \& Sussman, R. R. (1963). Ploidal inheritance in the slime mould Dictyostelium discoideum: haploidization and genetic segregation of diploid strains. J. gen. Microbiol. 30, 349. 